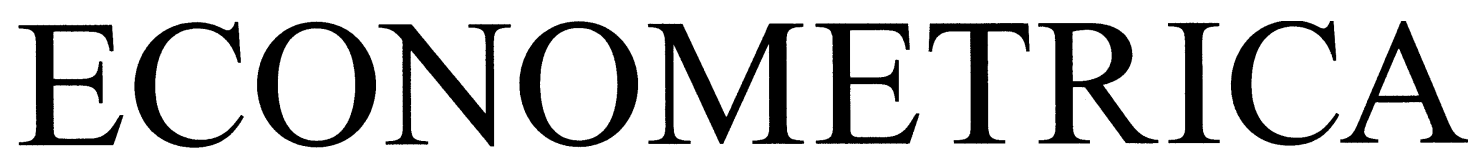

JOURNAL OF THE ECONOMETRIC SOCIETY

An International Society for the Advancement of Economic Theory in its Relation to Statistics and Mathematics

http://www.econometricsociety.org/

Econometrica, Vol. 78, No. 2 (March, 2010), 755-770

OBJECTIVE AND SUBJECTIVE RATIONALITY IN

A MULTIPLE PRIOR MODEL

ITZHAK GILBOA

HEC, Paris, France and Eitan Berglas School of Economics, Tel Aviv University, Tel Aviv 69978, Israel

FABIO MACCHERONI

Dondena, and IGIER, Università Bocconi, 20136 Milano, Italy

MASSIMO MARINACCI

Dondena, and IGIER, Università Bocconi, 20136 Milano, Italy

DAVID SCHMEIDLER

The Ohio State University, Columbus, OH 43210, U.S.A. and School of Mathematical Sciences, Tel Aviv University, Tel Aviv 69978, Israel

The copyright to this Article is held by the Econometric Society. It may be downloaded, printed and reproduced only for educational or research purposes, including use in course packs. No downloading or copying may be done for any commercial purpose without the explicit permission of the Econometric Society. For such commercial purposes contact the Office of the Econometric Society (contact information may be found at the website http://www.econometricsociety.org or in the back cover of Econometrica). This statement must be included on all copies of this Article that are made available electronically or in any other format. 


\title{
OBJECTIVE AND SUBJECTIVE RATIONALITY IN A MULTIPLE PRIOR MODEL
}

\section{By ITZHAK GILBOA, FABIO MACCHERONI, MASSIMO MARINACCI, AND DAVID SCHMEIDLER ${ }^{1}$}

\begin{abstract}
A decision maker (DM) is characterized by two binary relations. The first reflects choices that are rational in an "objective" sense: the DM can convince others that she is right in making them. The second relation models choices that are rational in a "subjective" sense: the DM cannot be convinced that she is wrong in making them.

In the context of decision under uncertainty, we propose axioms that the two notions of rationality might satisfy. These axioms allow a joint representation by a single set of prior probabilities and a single utility index. It is "objectively rational" to choose $f$ in the presence of $g$ if and only if the expected utility of $f$ is at least as high as that of $g$ given each and every prior in the set. It is "subjectively rational" to choose $f$ rather than $g$ if and only if the minimal expected utility of $f$ (with respect to all priors in the set) is at least as high as that of $g$. In other words, the objective and subjective rationality relations admit, respectively, a representation à la Bewley (2002) and à la Gilboa and Schmeidler (1989). Our results thus provide a bridge between these two classic models, as well as a novel foundation for the latter.
\end{abstract}

KEYWORDS: Multiple priors, rationality.

\section{INTRODUCTION}

A CENTRAL ISSUE IN DECISION UNDER UNCERTAINTY has been the modeling of Ellsberg-type phenomena (Ellsberg (1961)) that arise in the presence of ambiguity, that is, when decision makers (DMs) do not have enough information to quantify uncertainty with a single probability measure. Following Schmeidler's (1986, 1989) model involving nonadditive probabilities, Gilboa and Schmeidler (1989) and Bewley (2002) suggested models with sets of probabilities: the former modeling a complete preference relation by the maxmin rule, and the latter modeling an incomplete relation by the unanimity rule.

Our purpose here is to show that the two models, and their perspectives on ambiguity, are complementary and can be fruitfully combined in a preference formation perspective. We take a normative viewpoint and attempt to capture preferences that are justifiable. In many decision problems of interest, preferences that can be solidly justified are incomplete, yet decisions eventually have to be made. Thus we deal with two preference relations.

We correspondingly suggest two notions of rational choice. A choice is $o b$ jectively rational if the DM can convince others that she is right in making it.

\footnotetext{
${ }^{1}$ We wish to thank Eyal Baharad, Erio Castagnoli, Simone Cerreia-Vioglio, Eric Danan, Eddie Dekel, Gabi Gayer, Paolo Ghirardato, Al Klevorick, Dan Levin, Bob Nau, Klaus Nehring, Efe Ok, Wolfgang Pesendorfer, Ben Polak, Peter Wakker, and three anonymous referees for comments and references. This project was supported by the European Research Council (Advanced Grant BRSCDP-TEA), the Israel Science Foundation (Grants 975/03 and 355/06), and the Pinhas Sapir Center for Development.
} 
A choice is subjectively rational if others cannot convince the DM that she is wrong in making it. It can be useful to think of "being able to convince" as "having a proof that." Consider a model (not formalized here) in which preference statements such as $f \succsim g$ or $f \succ g$ are basic propositions, used to generate proofs in a given logic. A proof employs objective propositions that are accepted as statistical analysis of objective evidence, as scientific facts, and so forth, as well as preference statements by the DM. The proof is allowed to use standard logic, mathematical analysis, and statistical analysis, and also decision theoretic axioms. For example, transitivity may be used as an inference rule, allowing one to concatenate a proof that $f \succsim g$ with a proof that $g \succsim h$ to get a proof that $f \succsim h$. Using this logic metaphor, it is objectively rational to express a preference $f \succsim g$ if there is a proof that starts only with objective facts, and uses the logic described above to show that $f \succsim g$. In other words, one may view the objectively rational relation $\succsim$ as consisting of all provable preference statements. By contrast, the relation $\succsim$ is subjectively rational if, starting with its preference statements, no inconsistencies result. Thus, objective rationality is essentially a property of particular instances of the relation, whereas subjective rationality is a property of the entire relation. ${ }^{2}$

While we refer to objective and subjective rationality throughout the paper, the reader may think of an incomplete relation, describing justifiable choice, and a complete relation, describing the choices that will eventually be made.

To illustrate our approach, next we consider two classic axioms, that is, transitivity and independence. In this regard, observe that one may choose to model objective and subjective rationality using different axioms than those we employ in this paper. The axioms are supposed to capture the regularities satisfied by the two notions of rationality, that is, the ability to convince of or to insist on one's opinion. Which axioms are acceptable for these two notions is ultimately a subjective matter that may depend on culture or personality. Therefore, whether a particular axiom is satisfied by objective or subjective rationality becomes an empirical question, to be settled by the degree to which people tend to be convinced by arguments. It follows that the axioms we propose here should only be viewed as a suggestion for the list of regularities the two notions of rationality may satisfy.

\section{Transitivity}

We will require both the objective and the subjective rational relations to be transitive. However, this axiom is interpreted differently in the two cases.

\footnotetext{
${ }^{2}$ A more precise, though more cumbersome term for "objective rationality" would be "intersubjective rationality," because we do not make any reference to an externally defined objectivity or to "truth." Note that Simon $(1947,1957)$ also distinguished between objective rationality and subjective rationality, and in his case "objectivity" has a more classical meaning, referring to the experimenter's judgment.
} 
Regarding objective rationality, transitivity is a basic "inference rule," as explained above. When subjective rationality is discussed, we assume that all preference pairs are given as data. If the DM expresses strict preferences $f \succ g \succ h \succ f$, we believe that she can be convinced that her preferences are irrational or wrong. Assuming transitivity as a normative condition of subjective rationality captures this intuition.

\section{Independence}

We maintain that objective and subjective rationality differ when we consider the independence axiom as in Anscombe-Aumann's model, namely that $f \succsim g$ if and only if $\alpha f+(1-\alpha) h \succsim \alpha g+(1-\alpha) h$. Consider first objective rationality. Suppose that $f \succsim g$. Hence, there exists a proof, starting with objectively acceptable preferences, that $f$ is at least as good as $g$. The standard argument in favor of the independence axiom can be concatenated with this proof to conclude that $\alpha f+(1-\alpha) h \succsim \alpha g+(1-\alpha) h$.

Next suppose that subjective rationality is concerned. Assume that the DM expressed preferences $f \succsim g$ and $\alpha f+(1-\alpha) h \prec \alpha g+(1-\alpha) h$. Will she necessarily be embarrassed by these preferences? We maintain that this need not be the case. For example, assume that there are two states of the world, and that $f=(1,0)$ and $g=(0,1)$. The DM has no information about the probability of the two states, and therefore her objective rationality relation does not rank them. Having to make a decision, the DM might shrug her shoulders and decide that they are equivalent, namely, that $f \sim g$, due to symmetry. But when $f$ and $g$ are mixed with $h=f$ and $\alpha=1 / 2$, the mixture (1/2) $g+(1 / 2) h$ completely hedges against uncertainty, whereas the mixture of $f$ with itself does not provide any reduction of uncertainty. The DM might plausibly argue that in this case $\alpha f+(1-\alpha) h$ is not equivalent to $\alpha g+(1-\alpha) h$, because the former is uncertain, whereas the latter is not. As a result, the independence axiom is not as normatively appealing for subjective rationality as it is for objective rationality.

On the other hand, observe that no asymmetric uncertainty reduction would result if $f$ and $g$ were mixed with a constant act $h$. For this reason the conjunction of $f \succsim g$ and $\alpha f+(1-\alpha) h \prec \alpha g+(1-\alpha) h$ seems more difficult to justify as consistent, and subjective rationality will be required to satisfy $\mathrm{C}$ Independence.

\section{The Present Model}

We use two binary relations, $\left(\succsim^{*}, \succsim^{\wedge}\right)$, interpreted as objective and subjective rationality relations, respectively. We first provide a characterization of $\succsim^{*}$ so that it can be represented by a unanimity rule à la Bewley (2002) with a set of priors $C^{*}$ and a utility index $u^{*}$ (Theorem 1). If $\succsim^{\wedge}$ satisfies the axioms of Gilboa and Schmeidler (1989), then it can be represented by the maxmin rule 
with a set of priors $C^{\wedge}$ and a utility index $u^{\wedge}$ (Theorem 2). However, the two sets of priors and the two utility indexes are unrelated. We therefore introduce two additional properties, explicitly connecting the two relations.

The first property, Consistency, requires that a preference instance that is objectively rational is also subjectively rational. The second, Caution, deals with the way subjective rationality completes preferences between acts involving uncertainty and acts that do not. These two properties hold if and only if the two sets of priors $\left(C^{*}\right.$ and $\left.C^{\wedge}\right)$ are identical, and the two utility indexes $\left(u^{*}\right.$ and $u^{\wedge}$ ) are equivalent. Taken together, the axioms imply the existence of a set of priors and a utility index that represent both $\succsim^{*}$ and $\succsim^{\wedge}$ simultaneously: the former via unanimity and the latter via the maxmin rule (Theorem 3 ).

Finally, we observe that one of the connecting properties (Caution) guarantees uncertainty aversion. This suggests that the maxmin representation can follow even if the assumptions on $\succsim^{\wedge}$ are only completeness, transitivity, and continuity (Theorem 4).

\section{MODEL AND RESULTS}

\subsection{Preliminaries}

We use a version of the Anscombe and Aumann (1963) model as restated by Fishburn (1970). Let $X$ be a set of outcomes. A von Neumann-Morgenstern lottery is a finite support probability distribution over $X$. The set of lotteries, $L$, is endowed with a mixing operation: for every $P, Q \in L$ and every $\alpha \in[0,1]$, $\alpha P+(1-\alpha) Q \in L$ is defined pointwise (over $X$ ). The set of states of the world is $S$ endowed with an algebra $\Sigma$ of events. The set $\Delta(\Sigma)$ of (finitely additive) probabilities on $\Sigma$ is endowed with the eventwise convergence topology. The set of (simple) acts $F$ consists of all simple measurable functions $f: S \rightarrow L$. It is endowed with a mixture operation as well, performed pointwise (over $S$ ).

The DM is characterized by two binary relations $\succsim^{*}$ and $\succsim^{\wedge}$ on $F$, denoting objective and subjective rational preferences, respectively. The derived relations $\succ^{*}, \sim^{*}, \succ^{\wedge}$, and $\sim^{\wedge}$ are defined as usual. We extend $\succsim^{*}$ and $\succsim^{\wedge}$ to $L$ by identifying lotteries with constant acts. The set of all constant acts is denoted by $F_{c}{ }^{3}$

For a function $u: X \rightarrow \mathbb{R}$, and a lottery $P \in L$, we write, $\mathrm{E}_{P} u=\sum_{x \in X} P(x) u(x)$.

\subsection{Axioms}

We begin with the basic conditions that will be imposed on both relations $\succsim^{*}$ and $\succsim^{\wedge}$.

\footnotetext{
${ }^{3}$ We sometimes abuse notation by writing $P \in L$ for the corresponding constant act in $F_{c}$.
} 


\section{BASIC CONDITIONS:}

PREORDER: $\succsim$ is reflexive and transitive.

Monotonicity: For every $f, g \in F, f(s) \succsim g(s)$ for all $s \in S$ implies $f \succsim g$.

ARCHIMEDEAN CONTINUITY: For all $f, g, h \in F$, the sets $\{\lambda \in[0,1]: \lambda f+$ $(1-\lambda) g \succsim h\}$ and $\{\lambda \in[0,1]: h \succsim \lambda f+(1-\lambda) g\}$ are closed in $[0,1]$.

NONTRIVIALITY: There exist $f, g \in F$ such that $f \succ g$.

We interpret transitivity and monotonicity as axioms of rationality. Transitivity was discussed in the Introduction under the two interpretations. We here assume that monotonicity is also satisfied by the two relations: if act $f$ pointwise dominates act $g$, it should be easy for the DM to argue that $f$ is at least as good a choice as $g$; also, she will be embarrassed not to exhibit such a preference in this case.

Reflexivity is a model-related assumption: it states that we prefer to represent the weak rather than the strict part of the preference relations involved. In the presence of incomplete relations, such an assumption is not innocuous because strict preferences are not the complement of weak ones. Still, this is a modeling choice that makes no claims about the DM's preferences or behavior. Similarly, Nontriviality is a modeling assumption that simply rules out the uninteresting case of an overall indifferent DM, who would feature a constant utility function and any beliefs whatsoever, without any uniqueness results.

Finally, the continuity axiom has a familiar status: it can be viewed as a purely "technical" condition, having to do with the mathematical idealization we use, and it can also be viewed as an assumption whose content can be challenged by thought experiments.

Next we discuss axioms that are specific to objective or to subjective rationality. ${ }^{4}$

C-COMPleteness: For every $f, g \in F_{c}, f \succsim^{*} g$ or $g \succsim^{*} f$.

COMPLETENESS: For every $f, g \in F, f \succsim^{\wedge} g$ or $g \succsim^{\wedge} f$.

Observe that we require that objective rationality be complete when restricted to the subset of constant acts. C-Completeness verifies that the incom-

\footnotetext{
${ }^{4}$ Since each of the following axioms will be assumed for one relation only, we state them directly in terms of this relation rather than in terms of an abstract relation $\succsim$ as above. In the sequel, we use phrases such as "C-Completeness" and “ $\succsim *$ satisfies C-Completeness" interchangeably.
} 
pleteness of the objectively rational relation $\succsim^{*}$ is not due to any difficulties that the DM might have about determining her preferences under certainty. ${ }^{5}$

INDEPENDENCE: For every $f, g, h \in F$ and every $\alpha \in(0,1), f \succsim^{*} g$ if and only if $\alpha f+(1-\alpha) h \succsim^{*} \alpha g+(1-\alpha) h$.

C-INDEPENDENCE: For every $f, g \in F$, every $h \in F_{c}$, and every $\alpha \in(0,1), f \succsim^{\wedge}$ $g$ if and only if $\alpha f+(1-\alpha) h \succsim^{\wedge} \alpha g+(1-\alpha) h$.

UnCERTAINTY AVERSION: For every $f, g \in F$, if $f \sim^{\wedge} g$, then $(1 / 2) f+$ $(1 / 2) g \succsim^{\wedge} g$.

The two versions of the Independence axiom were discussed in the Introduction. Uncertainty Aversion is implied by Independence, and there is therefore no need to explicitly require that it holds for objective rationality. We find it a plausible assumption for subjective rationality due to the intuition that "smoothing out" acts should be desirable. That is, we assume that a DM who finds two acts, $f$ and $g$, equally attractive, will be embarrassed to state that their mixture is worse than both. Of course, this is but an assumption on what may or may not embarrass or convince the DM. For example, a DM who cannot reason in terms of the mixture operation may be subjectively rational while violating Uncertainty Aversion or C-Independence.

\subsection{Representation of Objective and of Subjective Rationality}

\subsubsection{Unanimity Representation of Objective Rationality}

The axioms we imposed on $\succsim^{*}$ deliver a unanimity representation. Our first result is conceptually similar to Bewley (2002). However, it represents a weak (rather than a strict) preference and it applies to an infinite state space; it is proved in Appendix B.

THEOREM 1: The following statements are equivalent:

(i) $\succsim^{*}$ satisfies the Basic Conditions, C-Completeness, and Independence.

(ii) There exist a nonempty closed and convex set $C^{*}$ of probabilities on $\Sigma$ and a nonconstant function $u^{*}: X \rightarrow \mathbb{R}$ such that, for every $f, g \in F$,

$$
f \succsim^{*} g \quad \text { iff } \quad \int_{S} \mathrm{E}_{f(s)} u^{*} d p(s) \geq \int_{S} \mathrm{E}_{g(s)} u^{*} d p(s) \quad \forall p \in C^{*} .
$$

Moreover, in this case, $C^{*}$ is unique and $u^{*}$ is unique up to positive affine transformations.

${ }^{5}$ Indeed, incompleteness of tastes will also result in incomplete preferences. See Aumann (1962), Kannai (1963), Richter (1966), Peleg (1970), and, more recently, Ok (2002), Dubra, Maccheroni, and Ok (2004), and Mandler (2005). Nau (2006) and Ok, Ortoleva, and Riella (2008) suggested models with incompleteness of both tastes and beliefs. 


\subsubsection{Maxmin Representation of Subjective Rationality}

The axioms we imposed on $\succsim^{\wedge}$ deliver a maxmin rule.

THEOREM 2-Gilboa and Schmeidler (1989, Theorem 1): The following statements are equivalent:

(i) $\succsim^{\wedge}$ satisfies the Basic Conditions, Completeness, C-Independence, and Uncertainty Aversion.

(ii) There exist a nonempty closed and convex set $C$ of probabilities on $\Sigma$ and a nonconstant function $u: X \rightarrow \mathbb{R}$ such that, for every $f, g \in F$,

$$
f \succsim^{\wedge} g \quad \text { iff } \quad \min _{p \in C} \int_{S} \mathrm{E}_{f(s)} u d p(s) \geq \min _{p \in C} \int_{S} \mathrm{E}_{g(s)} u d p(s) .
$$

Moreover, in this case, $C$ is unique and $u$ is unique up to positive affine transformations.

\subsection{Relating Objective and Subjective Rationality}

We now come to discuss the relationship between the two orders.

CONSISTENCY: $f \succsim^{*} g$ implies $f \succsim^{\wedge} g$.

Intuitively, we argued that it is subjectively rational to prefer $f$ to $g$ if the DM cannot be convinced that she is wrong in exhibiting such a preference. One way in which the DM can be proven wrong is by pointing out that there are compelling, objective reasons to exhibit the opposite preference. A similar condition appears in Nehring $(2001,2009)$, titled Compatibility. ${ }^{6}$ If $\left(\succsim^{*}, \succsim^{\wedge}\right)$ are represented as in Theorems 1 and 2, it is straightforward and essentially known that Consistency is equivalent to $u=u^{*}$ and $C \subseteq C^{*} .7$

CAUTION: For $g \in F$ and $f \in F_{c}, g \beth^{*} f$ implies $f \succsim^{\wedge} g$.

This property implies that the DM in question is rather averse to ambiguity. Comparing a potentially uncertain act $g$ and a constant (risky) act $f$, the DM first checks whether there are compelling reasons to prefer $g$ to $f$. If there are, namely, $g \succsim^{*} f$, the property has no bite (and $g \succsim^{\wedge} f$ would follow from Consistency). If, however, no such reasons can be found, the DM would opt for the risky act over the uncertain one.

Observe that Consistency appears to be essential to our interpretation: if the DM can be convinced of a given claim (i.e., that $f$ is at least as good as $g$ ), it

\footnotetext{
${ }^{6}$ In Nehring's work, compatibility is supposed to hold between a preference relation over acts, assumed complete, and a likelihood relation over events, allowed to be incomplete.

${ }^{7}$ See Nehring $(2001,2009)$ and Ghirardato, Maccheroni, and Marinacci (2004).
} 
should better be the case that she cannot be convinced that it is wrong to accept this claim. By contrast, Caution is a much more demanding assumption. If we impose it as a condition on subjective rationality, it suggests that, whenever $f$ is a constant act $\left(f \in F_{c}\right)$, the DM will be embarrassed to state a strict preference $g \succ f$ unless there is an "objective proof" that the weak preference $g \succsim f$ should hold. We discuss relaxations of this assumption below.

THEOREM 3: The following statements are equivalent:

(i) ${ }^{*}$ satisfies the Basic Conditions, C-Completeness, and Independence; ${ }^{\wedge}$ satisfies the Basic Conditions, Completeness, and C-Independence; and jointly ( $\left.\succsim^{*}, \succsim^{\wedge}\right)$ satisfy Consistency and Caution.

(ii) There exist a nonempty closed and convex set $C$ of probabilities on $\Sigma$ and a nonconstant function $u: X \rightarrow \mathbb{R}$ such that, for every $f, g \in F$,

$$
f \succsim^{*} g \quad \text { iff } \quad \int_{S} \mathrm{E}_{f(s)} u d p(s) \geq \int_{S} \mathrm{E}_{g(s)} u d p(s) \quad \forall p \in C
$$

and

$$
f \succsim^{\wedge} g \quad \text { iff } \quad \min _{p \in C} \int_{S} \mathrm{E}_{f(s)} u d p(s) \geq \min _{p \in C} \int_{S} \mathrm{E}_{g(s)} u d p(s) .
$$

Moreover, in this case, $C$ is unique and $u$ is unique up to positive affine transformations.

Notice that we do not need to assume that $\succsim^{\wedge}$ satisfies Uncertainty Aversion. In fact, its connection with $\succsim^{*}$ through Caution already guarantees that $\succsim^{\wedge}$ satisfies this property.

Theorem 3 can be also viewed as providing a novel foundation for the maxmin representation (2), based on the interplay of the two preferences ${ }^{*}$ and $\succsim^{\wedge}$ : the maxmin rule can be interpreted as a completion of the unanimity rule. If we take this approach, the following slightly stronger version of Caution allows us to further reduce the assumptions imposed on $\succsim^{\wedge}$.

Default to CERTAinty: For $g \in F$ and $f \in F_{c}, g \swarrow^{*} f$ implies $f \succ^{\wedge} g$.

This condition strengthens Caution by adding a "tie-breaking" rule that favors certainty. With this condition we can state another theorem.

THEOREM 4: Statements (i) and (ii) of Theorem 3 are equivalent to the following one:

(iii) ${ }^{*}$ satisfies the Basic Conditions, C-Completeness, and Independence; $\gtrsim^{\wedge}$ satisfies Preorder, Archimedean Continuity, and Completeness; and jointly ( ${ }^{*}$ , $\succsim^{\wedge}$ ) satisfy Consistency and Default to Certainty. 
This result can be interpreted as follows. Suppose that a DM starts with an incomplete preference relation, $\succsim^{*}$, satisfying the Basic Conditions, CCompleteness, and Independence, which thus admits a unanimity representation by a set of probabilities and a utility index. Suppose further that the DM needs to make decisions and that $\succsim^{\wedge}$ is her completion of $\succsim^{*}$, which also satisfies Preorder and Archimedean Continuity. Default to Certainty then characterizes a behavior (modeled by $\succsim^{\wedge}$ ) that conforms to the maxmin model. Thus, Theorem 4 provides another possible account by which maxmin behavior might emerge from incomplete preferences.

\section{DISCUSSION}

\subsection{Extremity of the Maxmin Rule}

The extreme nature of Caution is reflected in the extremity of the maxmin rule, when the set of probabilities $C$ is interpreted as representing "hard evidence." 8 Indeed, it has often been argued that evaluating an act $f$ by its worstcase expected utility is unreasonable. One may consider alternatives to Caution. Simply dropping the property allows a representation of $\succsim^{*}$ by one set of probabilities, $C^{*}$, as in (3), and a representation of $\succsim^{\wedge}$ by another set of probabilities, $C$, as in (4), where $C \subseteq C^{*}$. If $\succsim^{\wedge}$ satisfies Independence, $C$ will reduce to a singleton chosen out of $C^{*}$. Should one find this choice too arbitrary, one may choose a nonsingleton proper subset of $C^{*}$ as suggested, for example, by Gajdos, Hayashi, Tallon, and Vergnaud (2008). Another completion of $\succsim^{*}$ to a maxmin relation $\succsim^{\wedge}$ with a proper nonsingleton subset $C \subseteq C^{*}$ is suggested by Kopylov (2009), building on the present paper and the intuition of preference deferral suggested by Danan and Ziegelmeyer (2006). Finally, we mention that the set $C^{*}$ used for the unanimity rule may also be a subset of the set of probabilities defined by hard evidence. In fact, what counts as hard evidence is ultimately a subjective matter, as in the choice of significance level in hypotheses testing.

\subsection{Related Literature}

Ghirardato, Maccheroni, and Marinacci (GMM; 2004) modeled a preference relation $\succsim^{\wedge}$ which may exhibit nonneutrality to ambiguity, and they derived from it a relation that captures "unambiguous preferences." This relation, which they also denote by $\succsim^{*}$, is incomplete whenever $\succsim^{\wedge}$ fails to satisfy the independence axiom. Moreover, when $\succsim^{\wedge}$ is a maxmin expected utility relation, $\succsim^{*}$ turns out to be precisely the unanimity relation with respect to the same set of priors.

\footnotetext{
${ }^{8}$ However, the set $C$ in Gilboa and Schmeidler (1989) is derived from preferences and need not coincide with a set of probabilities that are externally given to the DM. The set $C$ is defined in behavioral terms, as a representation of a binary relation $\succsim^{\wedge}$, and it need not coincide with any cognitive notion of a set of probabilities.
} 
The present paper is close to GMM in terms of the mathematical structure, and we have indeed partly relied on its derivation of the unanimity rule (as opposed to the earlier work by Bewley (2002)). However, the emphasis is different. In our case, both $\succsim^{\wedge}$ and $\succsim^{*}$ are assumed as primitive relations, and the focus is on the relationships between them, as a step in the direction of modeling the reasoning process behind the completion of $\succsim^{*}$ to a subjectively rational but complete order $\succsim^{\wedge}$. If, for instance, one were to replace Caution by the requirement that $\succsim^{\wedge}$ satisfies independence, the derived relation $\succsim^{*}$ in GMM would equal $\succsim^{\wedge}$. By contrast, our model would still distinguish between subjective and objective rationality, and may be used to discuss the process by which a particular prior (corresponding to $\succsim^{\wedge}$ ) is selected out of the set of possible priors (corresponding to $\succsim^{*}$ ).

Nehring $(2001,2009)$ also discussed the tension between the inability to have complete preferences that are rationally derived and the need to make decisions. His model also deals with a pair of relations and the connection between them. In particular, he suggests that "contexts" can be used to choose a way to complete a relation, and has an axiom similar to our Consistency.

Formally, our unanimity representation result for $\succsim^{*}$, though independent, is similar to Girotto and Holzer (2005): the setup is slightly different and our proof is simpler.

There are other models that assume more than a single relation as a description of a preference formation process. Rubinstein (1988) discussed the generation of preferences over lotteries based on similarity relations. Mandler (2005) distinguished between "psychological preferences," which may be incomplete, and "revealed preferences," which are complete but may be intransitive. Danan (2008) also dealt with two relations, cognitive and behavioral.

\section{APPENDIX A: PRoOfS AND RELATEd MATERIAL}

$B_{0}(\Sigma)$ is the vector space generated by the indicator functions of the elements of $\Sigma$, endowed with the supnorm. We denote by ba( $\Sigma)$ the set of all bounded, finitely additive set functions on $\Sigma$, and by $\Delta(\Sigma)$ the set of all probabilities on $\Sigma$. As well known, ba $(\Sigma)$, endowed with the total variation norm, is isometrically isomorphic to the norm dual of $B_{0}(\Sigma)$. In this case the weak* topology, $w^{*}$, of ba $(\Sigma)$ coincides with the eventwise convergence topology.

Given a nonsingleton interval $K$ in the real line (whose interior is denoted $\left.K^{\circ}\right), B_{0}(\Sigma, K)$ is the set of the functions in $B_{0}(\Sigma)$ taking values in $K$. Clearly, $B_{0}(\Sigma)=B_{0}(\Sigma, \mathbb{R})$.

Proof OF THEOREM 3: Assume that $\left(\succsim^{*}, \succsim^{\wedge}\right)$ satisfy (i). Let $u^{*}$ and $C^{*}$ represent $\succsim^{*}$ as in Theorem 1 . In $F$, set $f \succsim^{\prime} g$ if and only if $\lambda f+(1-\lambda) h \succsim^{\wedge}$ $\lambda g+(1-\lambda) h$ for all $\lambda \in[0,1]$ and $h \in F$. Lemma 1 and Propositions 5 and 7 of GMM guarantee that there exist a nonempty closed and convex set $C$ of 
probabilities on $\Sigma$, a nonconstant function $u: X \rightarrow \mathbb{R}$, and a monotonic and constant linear functional $I: B_{0}(\Sigma) \rightarrow \mathbb{R}$ such that, for every $f, g \in F$,

$$
\begin{aligned}
& f \succsim^{\wedge} g \quad \text { iff } \quad I\left(\mathrm{E}_{f} u\right) \geq I\left(\mathrm{E}_{g} u\right), \\
& f \succsim^{\prime} g \quad \text { iff } \quad \int_{S} \mathrm{E}_{f(s)} u d p(s) \geq \int_{S} \mathrm{E}_{g(s)} u d p(s) \quad \forall p \in C, \\
& \min _{p \in C} \int_{S} \mathrm{E}_{f(s)} u d p(s) \leq I\left(\mathrm{E}_{f} u\right) .
\end{aligned}
$$

Moreover, equality holds in (7) for all $f \in F$ if (and only if) $\succsim^{\wedge}$ satisfies Uncertainty Aversion.

On constant acts, by Consistency, $\succsim^{*}$ (represented by E. $u^{*}$ ) is a nontrivial subrelation of $\succsim^{\wedge}$ (represented by E.u). Corollary B.3 of GMM allows us to assume $u^{*}=u$. Proposition 4 of GMM implies that $\succsim^{\prime}$ is the maximal subrelation of $\succsim^{\wedge}$ satisfying Independence. Consistency then implies $\succsim^{*} \subseteq \succsim^{\prime}$ and Proposition A.1 of GMM delivers $C \subseteq C^{*}$.

To show the converse inclusion, we use Caution. If there is $g \in F$ such that $I\left(\mathrm{E}_{g} u\right)>\min _{p \in C^{*}} \int_{S} \mathrm{E}_{g(s)} u d p(s)$, then there is $Q \in L$ such that $I\left(\mathrm{E}_{g} u\right)>$ $\mathrm{E}_{Q} u>\min _{p \in C^{*}} \int_{S} \mathrm{E}_{g(s)} u d p(s)$. That is, $g \not^{*} Q$ and $g \succ^{\wedge} Q$, which violates Caution. Thus, by (7) and $C \subseteq C^{*}, \min _{p \in C} \int_{S} \mathrm{E}_{f(s)} u d p(s) \leq I\left(\mathrm{E}_{f} u\right) \leq$ $\min _{p \in C^{*}} \int_{S} \mathrm{E}_{f(s)} u d p(s) \leq \min _{p \in C} \int_{S} \mathrm{E}_{f(s)} u d p(s)$ for all $f \in F$. Proposition A.1 of GMM delivers $C^{*} \subseteq C .{ }^{9}$ The rest is trivial.

Q.E.D.

ProOf OF THEOREM 4: Assume that ( $\left.\succsim^{*}, \succsim^{\wedge}\right)$ satisfy (iii). Let $C$ and $u$ represent $\succsim^{*}$ as in Theorem 1. Let $P, Q \in L$. By Consistency, $P \succsim^{*} Q$ implies $P \succsim^{\wedge} Q$. By Default to Certainty, $P \succ^{*} Q$ implies $P \succ^{\wedge} Q$. Therefore, $\succsim^{\wedge}$ and $\succsim^{*}$ coincide on $L$. Hence, Monotonicity of $\succsim^{*}$ and Consistency imply that $\succsim^{\wedge}$ also satisfies Monotonicity. Since $\succsim^{\wedge}$ satisfies Monotonicity and Archimedean Continuity, for each $f$ there exists $R_{f} \in L$ such that $R_{f} \sim^{\wedge} f$.

For every $f, f \beth^{*} R_{f}$ would imply, by Default to Certainty, $R_{f} \succ^{\wedge} f$. Hence, $f \succsim^{*} R_{f}$. Therefore, $\mathrm{E}_{R_{f}} u \leq \int_{S} \mathrm{E}_{f(s)} u d p(s)$ for all $p \in C$ and $\mathrm{E}_{R_{f}} u \leq$ $\min _{p \in C} \int_{S} \mathrm{E}_{f(s)} u d p(s)$.

Moreover, if $\mathrm{E}_{R_{f}} u<\min _{p \in C} \int_{S} \mathrm{E}_{f(s)} u d p(s)$, take $P \in L$ such that $P \succsim^{\wedge}$ $f(s)$ for all $s \in S$. Then there is $\gamma \in(0,1]$ such that $\mathrm{E}_{R_{f}} u<\mathrm{E}_{\gamma P+(1-\gamma) R_{f}} u=$ $\min _{p \in C} \int_{S} \mathrm{E}_{f(s)} u d p(s)$. Thus, $f \succsim^{*} \gamma P+(1-\gamma) R_{f} \succ^{\wedge} R_{f}$ and, by Consistency, $f \succ^{\wedge} R_{f}$, which is absurd. In conclusion, $\mathrm{E}_{R_{f}} u=\min _{p \in C} \int_{S} \mathrm{E}_{f(s)} u d p(s)$ for all $f \in F$ and all $R_{f} \in L$ such that $R_{f} \sim^{\wedge} f$.

Finally, $f \succsim^{\wedge} g \Longleftrightarrow R_{f} \succsim^{\wedge} R_{g} \Longleftrightarrow \mathrm{E}_{R_{f}} u \geq \mathrm{E}_{R_{g}} u \Longleftrightarrow$ $\min _{p \in C} \int_{S} \mathrm{E}_{f(s)} u d p(s) \geq \min _{p \in C} \int_{S} \mathrm{E}_{g(s)} u d p(s)$. The rest is trivial. Q.E.D.

\footnotetext{
${ }^{9}$ In particular, this implies that $\succsim^{\prime}$ coincides with $\succsim^{*}$.
} 


\section{APPENDIX B: SUPPLEMENTARY MATERIAL}

We recall that a binary relation $\gtrsim$ on $B_{0}(\Sigma, K)$ is:

- a preorder if it is reflexive and transitive,

- continuous if $\varphi_{n} \gtrsim \psi_{n}$ for all $n \in \mathbb{N}, \varphi_{n} \rightarrow \varphi$ and $\psi_{n} \rightarrow \psi$ imply $\varphi \gtrsim \psi$,

- Archimedean if the sets $\{\lambda \in[0,1]: \lambda \varphi+(1-\lambda) \psi \gtrsim \eta\}$ and $\{\lambda \in[0,1]: \eta \gtrsim$ $\lambda \varphi+(1-\lambda) \psi\}$ are closed in $[0,1]$ for all $\varphi, \psi, \eta \in B_{0}(\Sigma, K)$,

- affine if for all $\varphi, \psi, \eta \in B_{0}(\Sigma, K)$ and $\alpha \in(0,1), \varphi \gtrsim \psi$ if and only if $\alpha \varphi+$ $(1-\alpha) \eta \gtrsim \alpha \psi+(1-\alpha) \eta$

- monotonic if $\varphi \geq \psi$ implies $\varphi \gtrsim \psi$,

- nontrivial if there exists $\varphi, \psi \in B_{0}(\Sigma, K)$ such that $\varphi \gtrsim \psi$ but not $\psi \gtrsim \varphi$.

Proposition 1-GMM, Proposition A.1: For $j=1,2$, let $C_{j}$ be nonempty subsets of $\Delta(\Sigma)$ and let $\gtrsim_{j}$ be the relations defined on $B_{0}(\Sigma, K)$ by

$$
\varphi \gtrsim_{j} \psi \Longleftrightarrow \int_{S} \varphi d p \geq \int_{S} \psi d p \quad \forall p \in C_{j} .
$$

Then

$$
\varphi \gtrsim_{j} \psi \Longleftrightarrow \int_{S} \varphi d p \geq \int_{S} \psi d p \quad \forall p \in \overline{\mathrm{co}}^{w^{*}}\left(C_{j}\right)
$$

and the following statements are equivalent:

(i) $\varphi \gtrsim_{1} \psi \Longrightarrow \varphi \gtrsim_{2} \psi$ for all $\varphi$ and $\psi$ in $B_{0}(\Sigma, K)$.

(ii) $\overline{\mathrm{co}}^{w^{*}}\left(C_{2}\right) \subseteq \overline{\mathrm{co}}^{w^{*}}\left(C_{1}\right)$.

(iii) $\inf _{p \in C_{2}} \int_{S} \varphi d p \geq \inf _{p \in C_{1}} \int_{S} \varphi d p$ for all $\varphi \in B_{0}(\Sigma, K)$.

Proposition 2-GMM, Proposition A.2: 邓 is a nontrivial, continuous, affine, and monotonic preorder on $B_{0}(\Sigma, K)$ if and only if there exists a nonempty subset $C$ of $\Delta(\Sigma)$ such that

$$
\varphi \gtrsim \psi \quad \Longleftrightarrow \int_{S} \varphi d p \geq \int_{S} \psi d p \quad \forall p \in C .
$$

Moreover, $\overline{\mathrm{co}}^{w^{*}}(C)$ is the unique weak* closed and convex subset of $\Delta(\Sigma)$ representing $\gtrsim$ in the sense of Eq. (8).

The complete proofs of the above propositions appear in Ghirardato, Maccheroni, and Marinacci (2002). To prove our results, we need some additional lemmas.

LEMMA 1: Let $\gtrsim$ be a preorder on $B_{0}(\Sigma)$. Then $\gtrsim$ is affine if and only if $\varphi \gtrsim \psi$ implies $\gamma \varphi+\eta \gtrsim \gamma \psi+\eta$ for all $\eta \in B_{0}(\Sigma)$ and all $\gamma \in \mathbb{R}_{+}$.

The proof is a standard exercise. 
LEMMA 2: If $\gtrsim$ is an affine preorder on $B_{0}(\Sigma, K)$, then there exists a unique affine preorder $\gtrsim$ on $B_{0}(\Sigma)$ that coincides with $\gtrsim$ on $B_{0}(\Sigma, K)$. Moreover, if $\gtrsim$ is monotonic (resp. Archimedean), then $\gtrsim$ is monotonic (resp. Archimedean) too.

PROOF: Suppose first $0 \in K^{\circ}$. We begin with a claim:

CLAIM: Given any $\varphi, \psi \in B_{0}(\Sigma, K)$, the following facts are equivalent:

(i) $\varphi \gtrsim \psi$.

(ii) There exists $\alpha>0$ such that $\alpha \varphi, \alpha \psi \in B_{0}(\Sigma, K)$ and $\alpha \varphi \gtrsim \alpha \psi$.

(iii) $\alpha \varphi \gtrsim \alpha \psi$ for all $\alpha>0$ such that $\alpha \varphi, \alpha \psi \in B_{0}(\Sigma, K)$.

ProOF OF THE Claim: (i) $\Rightarrow$ (ii) and (iii) $\Rightarrow$ (i) are obvious. (ii) $\Rightarrow$ (iii) follows from affinity. Q.E.D.

If $\varphi, \psi \in B_{0}(\Sigma)$, set $\varphi \gtrsim^{\sharp} \psi \Longleftrightarrow \alpha \varphi \gtrsim \alpha \psi$ for some $\alpha>0$ such that $\alpha \varphi, \alpha \psi \in$ $B_{0}(\Sigma, K)$. By the Claim, $\gtrsim \sharp$ is a well defined binary relation on $B_{0}(\Sigma)$, which coincides with $\gtrsim$ on $B_{0}(\widetilde{\Sigma}, K)$. Moreover, $\varphi \gtrsim^{\sharp} \psi$ if and only if $\alpha \varphi \gtrsim \alpha \psi$ for all $\alpha>0$ such that $\alpha \varphi, \alpha \psi \in B_{0}(\Sigma, K)$. By standard arguments, $\gtrsim \sharp$ is an affine preorder and it is monotonic if $\gtrsim$ is monotonic.

As to uniqueness, let $\gtrsim^{b}$ be an affine preorder on $B_{0}(\Sigma)$ that coincides with $\gtrsim$ on $B_{0}(\Sigma, K)$. For all $\varphi, \psi \in B_{0}(\Sigma)$, take $\alpha>0$ such that $\alpha \varphi, \alpha \psi \in B_{0}(\Sigma, K)$. Then the Claim (applied to $\left.\gtrsim^{b}\right)$, the fact that $\gtrsim^{b}$ coincides with $\gtrsim$ on $B_{0}(\Sigma, K)$, and the definition of $\gtrsim^{\sharp}$ guarantee that $\varphi \gtrsim^{b} \psi \Longleftrightarrow \alpha \varphi \gtrsim^{b} \alpha \psi \Longleftrightarrow \alpha \varphi \gtrsim$ $\alpha \psi \Longleftrightarrow \varphi \gtrsim^{\sharp} \psi$, that is, $\gtrsim^{b}$ coincides with $\gtrsim^{\sharp}$ on $B_{0}(\Sigma)$.

Suppose $0 \notin K^{\circ}$. Given any $k \in K^{\circ}$, for $\varphi, \psi \in B_{0}(\Sigma, K-k)$ set $\varphi \gtrsim_{k} \psi \Longleftrightarrow$ $\varphi+k \gtrsim \psi+k$. Then $\gtrsim_{k}$ is an affine preorder on $B_{0}(\Sigma, K-k)$ (monotonic if $\gtrsim$ is monotonic). Since $0 \in(K-k)^{\circ}$, there is a unique affine preorder $\gtrsim_{k}^{\sharp}$ on $B_{0}(\Sigma)$ that coincides with $\gtrsim_{k}$ on $B_{0}(\Sigma, K-k)$ (monotonic if $\gtrsim$ is monotonic). Such an extension coincides with $\gtrsim$ on $B_{0}(\Sigma, K)$ and it is the unique affine preorder on $B_{0}(\Sigma)$ with this property.

Finally, if $\gtrsim$ is Archimedean, denote by $\gtrsim$ the unique affine preorder on $B_{0}(\Sigma)$ which coincides with $\gtrsim$ on $B_{0}(\Sigma, K)$. For all $\varphi, \psi, \eta \in B_{0}(\Sigma)$ take $\alpha>0$ and $\beta \in \mathbb{R}$ such that $\alpha \varphi+\beta, \alpha \psi+\beta, \alpha \eta+\beta \in B_{0}(\Sigma, K)$. Then, by Lemma 1 , $\left\{\lambda \in[0,1]: \lambda \varphi+(1-\lambda) \psi \gtrsim^{\sharp} \eta\right\}$ coincides with $\{\lambda \in[0,1]: \lambda(\alpha \varphi+\beta)+(1-$ $\lambda)(\alpha \psi+\beta) \gtrsim \alpha \eta+\beta\}$, which is closed since $\gtrsim$ is Archimedean. This argument and the one obtained by reversing all the relations show that $\gtrsim \sharp$ is Archimedean.

Q.E.D.

LEMMA 3: An affine and monotonic preorder $\gtrsim$ on $B_{0}(\Sigma, K)$ is continuous if and only if it is Archimedean.

ProOF: Obviously, continuity implies the Archimedean property. Conversely, assume $\gtrsim$ is Archimedean. Since $\gtrsim$ is monotonic and Archimedean, then the affine preorder $\gtrsim$ on $B_{0}(\Sigma)$ that coincides with $\gtrsim$ on $B_{0}(\Sigma, K)$ is monotonic and Archimedean too (Lemma 2). 
If $\varphi_{n} \gtrsim^{\sharp} 0$ for all $n \in \mathbb{N}$ and $\varphi_{n} \rightarrow \varphi$, let $M=\sup _{s \in S} \varphi(s)$, which is indeed a maximum. For all $\varepsilon \in(0,1)$ there is $n$ such that $\varphi_{n} \leq \varphi+\varepsilon \leq \varphi+\varepsilon(M+$ $1-\varphi)$. In fact, $M \geq \varphi$ implies $M+1-\varphi \geq 1$. Therefore, for all $\varepsilon \in(0,1)$ there is $n \in \mathbb{N}$ such that $\varepsilon(M+1)+(1-\varepsilon) \varphi=\varphi+\varepsilon(M+1-\varphi) \geq \varphi_{n} \gtrsim^{\sharp} 0$. Monotonicity of $\gtrsim^{\sharp}$ delivers that, for all $\varepsilon \in(0,1), \varepsilon(M+1)+(1-\varepsilon) \varphi \gtrsim^{\sharp} 0$, but $\gtrsim$ is Archimedean, hence the set of all $\varepsilon$ with this property is closed and because it contains $(0,1)$, it also contains 0 ; in particular, $\varphi \gtrsim^{\sharp} 0$.

Conclude that, if $\varphi_{n} \rightarrow \varphi, \psi_{n} \rightarrow \psi$, and $\varphi_{n} \gtrsim^{\sharp} \psi_{n}$ for all $n \in \mathbb{N}$, then $\varphi_{n}-$

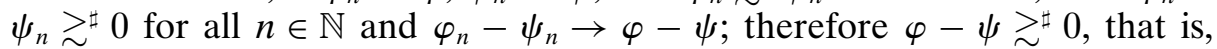
$\varphi \gtrsim^{\sharp} \psi$. Thus, $\gtrsim^{\sharp}$ is continuous, which immediately implies that $\gtrsim \underset{\text { is continuous }}{ }$ too.

Q.E.D.

Now Lemma 3 and Proposition 2 deliver the following corollary.

COROLlaRY 1: $\gtrsim$ is a nontrivial, Archimedean, affine, and monotonic preorder on $B_{0}(\Sigma, K)$ if and only if there exists a nonempty subset $C$ of $\Delta(\Sigma)$ such that

$$
\varphi \gtrsim \psi \quad \Longleftrightarrow \int_{S} \varphi d p \geq \int_{S} \psi d p \quad \forall p \in C .
$$

Moreover, $\overline{\mathrm{co}}^{w^{*}}(C)$ is the unique weak ${ }^{*}$ closed and convex subset of $\Delta(\Sigma)$ representing $\gtrsim$ in the sense of $E q$. (9).

All the results we have proved in this appendix hold more generally if $B_{0}(\Sigma)$ is replaced by any normed Riesz space with unit. ${ }^{10}$

Proof of THEOREM 1: Assume (i). By standard arguments, there exists a nonconstant $u^{*}$ such that $U(\cdot) \equiv$ E. $u^{*}$ represents $\succsim^{*}$ on $L$. Also, $B_{0}(\Sigma, U(L))=$ $\{U \circ f: f \in F\}$ and $U \circ f=U \circ g$ if and only if $f(s) \sim^{*} g(s)$ for all $s \in S$, which by Monotonicity implies $f \sim^{*} g$. We can therefore define $\gtrsim^{*}$ as follows: for $\varphi, \psi \in B_{0}(\Sigma, U(L)), \varphi \gtrsim^{*} \psi$ if $f \succsim^{*} g$ for some $f, g \in F$ such that $\varphi=U \circ f$ and $\psi=U \circ g$.

By standard arguments, (i) implies that $\gtrsim^{*}$ is a preorder that satisfies the conditions of Corollary 1 . Hence, there exists a unique nonempty weak* closed and convex subset $C^{*}$ of $\Delta(\Sigma)$ such that, for $\varphi, \psi \in B_{0}(\Sigma, U(L)), \varphi \gtrsim^{*} \psi \Longleftrightarrow$ $\int_{S} \varphi d p \geq \int_{S} \psi d p$ for all $p \in C^{*}$. Therefore, for $f, g \in F, f \gtrsim^{*} g \Longleftrightarrow U \circ f \gtrsim^{*}$ $U \circ g \Longleftrightarrow \int_{S}(U \circ f) d p \geq \int_{S}(U \circ g) d p$ for all $p \in C^{*} \Longleftrightarrow \int_{S} \mathrm{E}_{f(s)} u^{*} d p(s) \geq$ $\int_{S} \mathrm{E}_{g(s)} u^{*} d p(s)$ for all $p \in C^{*}$. The rest is trivial.

Q.E.D.

Final Technical Remarks

REMARK 1: There is a natural trade-off between Archimedean Continuity and Independence. Theorem 1 holds unchanged if we replace Archimedean

\footnotetext{
${ }^{10}$ For definitions, see Chapter 8 of Aliprantis and Border (2006).
} 
Continuity with the following stronger condition and replace Independence with the following weaker condition.

STRONG ARChimedeAn CONTINUITy: For all $e, f, g, h \in F$, the set $\{\lambda \in$ $\left.[0,1]: \lambda f+(1-\lambda) g \succsim^{*} \lambda h+(1-\lambda) e\right\}$ is closed in $[0,1]$.

WEAK INDEPENDENCE: For every $f, g, h \in F$, and every $\alpha \in(0,1), f \succsim^{*}$ $g$ implies $\alpha f+(1-\alpha) h \succsim^{*} \alpha g+(1-\alpha) h$.

In fact, Strong Archimedean Continuity implies Archimedean Continuity, while Shapley and Baucells (1998, Lemma 1.2) showed that Preorder, Strong Archimedean Continuity, and Weak Independence imply Independence. Thus, representation (1) holds if Archimedean Continuity and Independence are replaced by Strong Archimedean Continuity and Weak Independence. Conversely, (1) implies Strong Archimedean Continuity and (Weak) Independence.

REMARK 2: Lemma 3 and the implied Corollary 1 are the main technical novelties, with respect to the results of GMM, that we need for the proof of Theorem 1. Lacking the link between the algebraic Archimedean property and the topological continuity property established here, GMM had to resort to the topological continuity of the functional that represented the original preferences so as to obtain a unanimity representation of the derived unambiguous preferences (see the proof of their Proposition 5). As already observed, here $\succsim^{*}$ is assumed as a primitive and their techniques cannot be directly replicated.

\section{REFERENCES}

Aliprantis, C. D., AND K. C. Border (2006): Infinite Dimensional Analysis (Third Ed.). New York: Springer-Verlag. [768]

Anscombe, F. J., AND R. J. Aumann (1963): “A Definition of Subjective Probability,” The Annals of Mathematics and Statistics, 34, 199-205. [758]

Aumann, R. J. (1962): "Utility Theory Without the Completeness Axiom," Econometrica, 30, 445-462. [760]

Bewley, T. (2002): "Knightian Decision Theory: Part I," Decisions in Economics and Finance, 25, 79-110. [755,757,760,764]

DANAN, E. (2008): "Revealed Preference and Indifferent Selection," Mathematical Social Sciences, 55, 24-37. [764]

Danan, E., AND A. Ziegelmeyer (2006): "Are Preferences Complete? An Experimental Measurement of Indecisiveness Under Risk," Working Paper, Max Planck Institute of Economics. [763]

DUbra, J., F. MACChERONI, AND E. A. OK (2004): “Expected Utility Theory Without the Completeness Axiom," Journal of Economic Theory, 115, 118-133. [760]

ELLSBERG, D. (1961): "Risk, Ambiguity and the Savage Axioms," Quarterly Journal of Economics, 75, 643-669. [755]

FISHBURN, P. C. (1970): Utility Theory for Decision Making. New York: Wiley. [758]

Gajdos, T., T. HAYASHI, J.-M. TALLON, AND J.-C. VergnaUd (2008): "Attitude Toward Imprecise Information," Journal of Economic Theory, 140, 27-65. [763] 
Ghirardato, P., F. MACCheroni, AND M. MarinaCCI (2002): "Ambiguity From the Differential Viewpoint," Social Science Working Paper 1130, Caltech. [766]

(2004): "Differentiating Ambiguity and Ambiguity Attitude," Journal of Economic Theory, $118,133-173 .[761,763]$

GILBOA, I., AND D. SCHMEIDLER (1989): "Maxmin Expected Utility With Non-Unique Prior," Journal of Mathematical Economics, 18, 141-153. [755,757,761,763]

GirotTo, B., AND S. HOLZER (2005): "Representation of Subjective Preferences Under Ambiguity," Journal of Mathematical Psychology, 49, 372-382. [764]

KANNAI, Y. (1963): "Existence of a Utility in Infinite Dimensional Partially Ordered Spaces," Israel Journal of Mathematics, 1, 229-234. [760]

KopYLOV, I. (2009): "Choice Deferral and Ambiguity Aversion," Theoretical Economics, 4, 199-225. [763]

MANDLER, M. (2005): "Incomplete Preferences and Rational Intransitivity of Choice," Games and Economic Behavior, 50, 255-277. [760,764]

NAU, R. (2006): "The Shape of Incomplete Preferences," The Annals of Statistics, 34, 2430-2448. [760]

Nehring, K. (2001): "Ambiguity in the Context of Probabilistic Beliefs," Mimeo, UC Davis. $[761,764]$

[2009): "Imprecise Probabilistic Beliefs as a Context for Decision-Making Under Ambiguity," Journal of Economic Theory, 144, 1054-1091. [761,764]

OK, E. A. (2002): "Utility Representation of an Incomplete Preference Relation," Journal of Economic Theory, 104, 429-449. [760]

OK, E. A., P. ORTOLEVA, AND G. Riella (2008): "Incomplete Preferences Under Uncertainty: Indecisiveness in Beliefs vs. Tastes," Mimeo, NYU. [760]

PeleG, B. (1970): "Utility Functions for Partially Ordered Topological Spaces," Econometrica, 38, 93-96. [760]

RICHTER, M. (1966): "Revealed Preference Theory,” Econometrica, 34, 625-645. [760]

RUBINSTEIN, A. (1988): "Similarity and Decision-Making Under Risk," Journal of Economic Theory, 46, 145-153. [764]

SCHMEIDLER, D. (1986): "Integral Representation Without Additivity," Proceedings of the American Mathematical Society, 97, 255-261. [755]

(1989): "Subjective Probability and Expected Utility Without Additivity," Econometrica, 57, 571-587. [755]

ShaPley, L. S., AND M. BAUCELls (1998): "A Theory of Multiperson Utility,” Working Paper 779, UCLA. [769]

SIMON, H. A. (1947): Administrative Behavior (Second Ed.). New York: The Free Press. [756]

(1957): "Comparison of Game Theory and Learning Theory," in Models of Man. New York: Wiley, 274-279. [756]

HEC, Paris, France and Eitan Berglas School of Economics, Tel Aviv University, Tel Aviv 69978, Israel; tzachigilboa@gmail.com,

Department of Decision Sciences, Dondena, and IGIER, Università Bocconi, 20136 Milano, Italy; fabio.maccheroni@unibocconi.it,

Department of Decision Sciences, Dondena, and IGIER, Università Bocconi, 20136 Milano, Italy; massimo.marinacci@unibocconi.it, and

Department of Economics, The Ohio State University, Columbus, OH 43210, U.S.A. and School of Mathematical Sciences, Tel Aviv University, Tel Aviv 69978, Israel; schmeidler.1@osu.edu.

Manuscript received November, 2008; final revision received September, 2009. 